\title{
ITM2B amyloidosis
}

INSERM

\section{Source}

INSERM. (1999). Orphanet: an online rare disease and orphan drug data base. ITM2B amyloidosis. ORPHA:439254

A rare, neurodeg enerative disease characterized by progressive dementia and ataxia, widespread cerebral amyloid angiopathy and parenchymal amyloid deposition. Two subtypes have been identified, ABri amyloidosis and ADan amyloidosis. 